\title{
Gestión del capital intelectual (cI) dinámico en la hotelería, una reflexión teórica
}

Recibido: 16/09/2016 · Aceptado: 21/02/2017

\author{
Carlos Hugo Millán-García* \\ Facultad de Economía \\ María del Rocío Gómez-Díaz \\ Facultad de Contaduría y Administración \\ Elva Esther Vargas Martínez \\ Facultad de Turismo y Gastronomía \\ Universidad Autónoma del Estado de México
}

\section{Resumen}

En la denominada economía del conocimiento, el capital intelectual (ci) adquiere gran importancia para la generación de ventajas competitivas en las empresas, particularmente para las del sector hotelero, ya que en muchas de ellas las limitaciones para elevar su competitividad se deben, entre otros aspectos, a altas tasas de rotación de personal, falta de aprovechamiento de conocimientos y bajo manejo de información de manera sistemática. En esta investigación se presenta una revisión de estudios que reconocen la importancia del cr y el avance e impacto que ha producido, hasta la propuesta de una teoría dinámica del cr, referida a su empleo en las empresas con el objetivo de identificar activos del capital intelectual factibles de ser aprovechados por la hotelería en procesos de gestión, para transitar de un estatus estático a uno dinámico y con ello potenciar su competitividad. Los principales resultados muestran que la sistematización de procesos para aprovechar el potencial del capital humano (cH) y el cúmulo de conocimientos que radica en él, los mecanismos que favorecen la generación de información a partir del capital relacional (CR), y la implementación de sistemas y aprovechamiento de la tecnología para consolidar el capital estructural (CE) permitirán, bajo una aplicación estratégica, contribuir a elevar la competitividad en las empresas del ramo de la hotelería.

Palabras clave: Capital intelectual, humano, estructural, relacional, hotelería.

*Correos electrónicos: chmillang@uaemex.mx, rgomezd44@hotmail.com, elvacolegio@hotmail.com

TeORía y Praxis · ISSN 18701582 · NÚM. 22 · MAYO-AGOSTO 2017 · pp. 85-112 


\title{
Management of dynamic intellectual capital (cI) in the hotel industry, a theoretical reflection
}

Recieved: 16/09/2016 · Accepted: 21/02/2017

\author{
Carlos Hugo Millán-García* \\ Facultad de Economía \\ María del Rocío Gómez-Díaz \\ Facultad de Contaduría y Administración \\ Elva Esther Vargas Martínez \\ Facultad de Turismo y Gastronomía \\ Universidad Autónoma del Estado de México
}

\begin{abstract}
In the so called knowledge economy, intellectual capital (Ic) is of great importance for the generation of competitive advantages in companies, particularly in those of the hotel sector, since in many of them the limitations to increase their competitiveness are caused by, among others, high rates of staff turnover; lack of knowledge skills and low systematic use of information. In this research a review of studies that recognize the importance of Ic as well as it's progress and generated impact are presented. A proposal of a dynamic theory of ic is included referring to the way it is used in companies with the objective of identifying assets of intellectual capital that are feasible to be taking advantage of in the hotel industry, applying them in management processes to move from a static to a dynamic status and thus enhancing their results in terms of competitiveness. The main results show that the systematization of processes to take advantage of the potential of human capital (Hc) and the accumulation of knowledge that resides within, the mechanisms that favor the generation of information from the relational capital (rc); and the implementation of systems and use of technology to consolidate the structural capital (sc) will allow, under a strategic application, to contribute to increase the competitiveness in companies in the hotel sector.
\end{abstract}

KEY worDs: Intellectual capital, human, structural, relational, hotel industry.

*E-mails: chmillang@uaemex.mx, rgomezd44@hotmail.com,elvacolegio@hotmail.com 


\section{Introducción}

La globalización y el ritmo acelerado en el desarrollo de las tecnologías de la información han provocado condiciones en el mercado (competidores, clientes, proveedores, sociedad) que impulsan a las empresas a gestionar de mejor manera sus recursos en la búsqueda de mayor competitividad, entendida como una forma integral más allá de la obtención de beneficios económicos inmediatos (Manosalvas y Manosalvas, 2014; Saavedra, 2012).

El Foro Económico Mundial expuso que la clave para la competitividad de los países y las organizaciones radica en las capacidades, habilidades y talentos de las personas que los integran (World Economic Forum, 2013).

En este contexto, una gestión estratégica en empresas hoteleras respaldada con la valoración y aplicación del capital intelectual (cI) y sus componentes es de vital observancia para la industria turística en general y la hotelería en particular, ya que el mercado demanda su diferenciación ante la gran oferta de productos, lo cual provoca que sus ciclos de vida sean más cortos y que los precios ya no marquen diferencia (Bravo, Andrade y Delgado, 2014), pues en la actualidad los turistas se tornan con mayor experiencia e información exigiendo la inclusión y aprovechamiento de la tecnología, personalización y flexibilidad de los servicios turísticos (Lillo, Ramón y Sevilla, 2007).

Así, el reto consiste en la búsqueda de activos del cr que las empresas puedan implementar como parte de su estrategia (Marzo y Scarpino, 2016; Zambon, 2016; Aisenberg, Durst, Zaniboni y Selig, 2015; Dumay y Garanina, 2013), además de hacer operativos los constructos teóricos que mejoren su desempeño (Aisenberg et al., 2015; Dumay y Garanina, 2013), ya que se señala que a pesar de toda la información que se tiene a la mano, en el nivel empresarial todavía no se sabe "cómo hacerlo, y lo que es peor, no saben si su hoja de ruta es correcta, al carecer de referenciales para lograr su objetivo” (López y Nevado, 2016, p. 9).

El presente estudio forma parte de una investigación que pretende analizar las relaciones entre el cr y la competitividad en la hotelería. Este artículo corresponde a la revisión de literatura en la que se identifica la necesidad de transitar del cr estático hacia un estatus dinámico, lo que puede llevarse a cabo por medio de la sistematización administrativa, tecnológica y de información. 
Con base en lo anterior, como resultado se proponen estrategias teniendo en cuenta activos intangibles claves de la hotelería, para que a través de su gestión colaboren en el incremento de ventajas competitivas

El documento se estructura en tres secciones: la primera corresponde a la fundamentación teórica, la evolución del cı desde su taxonomía hacia su medición y reporte; la necesidad de la transición del cr estático al cr dinámico; y los referentes contextuales que vinculan la hotelería con el cr; la segunda sección describe el desarrollo metodológico que sustenta la investigación, y la tercera se propone acciones y estrategias para que el ci pueda ser capitalizado en la hotelería en su modalidad dinámica, finalizando con las conclusiones y referencias consultadas.

\section{Marco de fundamentación}

Las empresas del sector hotelero, al igual que las comerciales y de servicios, enfrentan en la actualidad el reto de lograr mayor penetración en el mercado, para lo cual requieren incorporar prácticas de manera cotidiana que las lleven a alcanzar mayores niveles de competitividad. Así, el cı puede constituir un instrumento de apoyo ante este reto.

\section{Reconocimiento e importancia del cI}

A diferencia de las sociedades agrícolas, donde el principal recurso era la tierra, y de las industriales, que era el capital físico, en la actualidad, el factor estratégico para las empresas es el conocimiento (Bueno, Salmador y Merino, 2008; Mouritsen, 2006); por ello se habla de una nueva era y de una economía del conocimiento (Edvinsson, 1997, p. 366).

De esta concepción se desprende que el verdadero valor de las empresas se encuentra en sus activos intangibles, una serie de elementos que permanecen ocultos de los balances financieros y que incrementan su valor no solo en el momento actual, sino también hacia el futuro. Es decir, que el valor financiero de las empresas y el que tienen en el mercado no es el mismo, pues el segundo resulta ser superior al primero, y esa diferencia entre ambos es atribuida al CI (Edvinsson, 1997). 
Autores como Marzo y Scarpino (2016), Inkinen (2015), Berzkalne y Zelgalve (2014), y Mercado, Nava y Demuner (2012) destacan la relevancia y valor de estos intangibles, reconociéndolos como la principal fuente de ventaja competitiva empresarial cuando se pretende contender en los mercados globales. Esa conciencia sobre la presencia e importancia del cr en las empresas ha dado pie a su estudio y análisis, primeramente buscando definirlo, aunque a la fecha no se ha logrado alcanzar un consenso (Giuliani, 2015; Ienciu y Matiş, 2012). No obstante, en los puntos coincidentes se hace referencia a "la posesión de conocimiento, la experiencia aplicada, la tecnología organizacional, la relación con los clientes y las habilidades profesionales que proveen a la empresa una ventaja competitiva en el mercado" (Edvinsson, 1997, p. 368). Asimismo, existen autores que refieren al cr como el conjunto de activos intangibles que las organizaciones poseen y que por su naturaleza no se reflejan en los estados financieros (Ienciu y Matiş, 2012; Berzkalne y Zelgalve, 2014), que incluyen los conocimientos y competencias que les pueden aportar valor y ventajas competitivas en el futuro (Inkinen, 2015; López y Nevado, 2006).

A pesar de la dispersión de definiciones es notoria su asociación con las siguientes características: no estar reflejados en los estados financieros de las empresas, pero que tienen valor y lo aportan a ellas; son considerados activos y como tales proyectan a estas hacia un mejor desempeño futuro; radican en las propias organizaciones y se encuentran depositados en ellas y su personal; son obtenidos y acumulados en las habilidades, conocimientos, métodos, experiencias, procesos, relaciones, comunicación, documentos, marcas, patentes, bases de datos, entre otros (Inkinen, 2015; Ienciu y Matiş, 2012; Bueno et al., 2008; López y Nevado, 2006; Engström, Westnes y Westnes, 2003; Edvinsson, 1997).

A partir de la identificación de los activos intangibles presentes en las empresas, se comienza con su clasificación. Edvinsson (1997), por ejemplo, en una de las primeras propuestas reconoció para la empresa Skandia AFS que sus componentes pueden conformar una lista interminable, agrupándolos en capital humano ( $\mathrm{CH}$ ) y capital estructural ( $\mathrm{CE}$ ), partiendo de que el cr es básicamente conocimiento, experiencia aplicada, tecnología organizacional, relación con clientes y habilidades profesionales (Edvinsson, 1997, p. 368); considera además que, de esos elementos, las empresas son dueñas del cE, no así del ch, que solo lo renta. También hace alusión a otros capitales (como clientes, organizacional, innovación, procesos), pero como subdivisiones del CE. 
En los últimos años se han realizado esfuerzos por identificar la taxonomía del ci (Marzo y Scarpino, 2016; Aisenberg et al., 2015; Dumay y Garanina, 2013) debido a que la clasificación no se consideraba de aplicación general, lo cual dio como resultado la proliferación y reacomodo de esquemas que particularizan las características propias de sectores e industrias diversas, llegándose a encontrar hasta nueve componentes distintos, algunos redundantes entre sí (Inkinen, 2015; Aisenberg et al., 2015). Inkinen (2015), en su estudio, encuentra la divergencia y convergencia de ellos, así como el sentido que le ha sido dado por los diferentes autores que los proponen (véase cuadro 1); identifica que el capital humano es aquel perteneciente a la inteligencia de los miembros de la organización y se constituye por todos aquellos atributos que derivan de las habilidades y conocimientos que las empresas utilizan a través de los empleados, y considera que este elemento puede ser el principal dentro del cr. También señala que el capital organizacional (co) y el cE se han usado como términos homólogos para aludir a los elementos de la empresa que dan soporte al desempeño de los empleados, por ejemplo la forma de organización, las estructuras, los procesos y las rutinas, entre otros (Inkinen, 2015).

Algo similar ocurre con el capital relacional, el capital clientes y el capital social, pues para hacer referencia al primero se engloba el conocimiento que radica en las relaciones que se establecen con cualquiera de los agentes externos a la empresa, mientras que el segundo y el tercero separan, por un lado, las relaciones con los clientes y canales de distribución (capital clientes) y, por el otro, aquellas con las personas o grupos de interés del entorno (capital social) (Inkinen, 2015).

El capital innovación es concebido como la habilidad de la empresa para transformar o utilizar conocimientos ya existentes en ella, enfocado en crear nuevos productos, procesos y tecnología, que pueden englobarse como elementos del CE al formar parte de la propiedad intelectual o que puede ser visto en cuanto actividad de gestión del cr, según se observará más adelante.

El capital información y el capital tecnológico los califica como los sistemas empleados por las organizaciones para aprovechar la información como recurso, y el uso de la tecnología para esos mismos sistemas de información, aunque también se pueden considerar elementos del CE. 
Cuadro 1. Componentes del capital intelectual (cI) empleados en la literatura y componentes básicos
Componentes identificados por Inkinen en diversos estudios

Capital Humano

Capital Relacional Capital Clientes

Capital Social

Capital Organizacional

Capital Estructural

Capital Innovación

Capital Información

Capital Tecnológico
Componente

básico al que pertenece

Capital

Humano

Capital

Relacional

Capital

Estructural
Características

Habilidades, conocimientos, competencia, actitud, habilidad intelectual, capacidades, educación, satisfacción, valores, creatividad, trabajo en equipo, resolución de problemas, habilidades de comunicación, liderazgo, confianza.

El valor y conocimiento obtenido de las relaciones de las empresas con el exterior, en todas sus dimensiones como lo son clientes, proveedores, distribuidores, socios, comunidad local y otras entidades relacionadas como el gobierno

Estructuras, procesos, rutinas, sistemas de información, documentos, bases de datos, planes, propiedad intelectual, estrategia de la organización, cultura organizacional, filosofía empresarial, marcas, logotipos, sistemas, patentes, secretos industriales, innovación, ambiente laboral.

Fuente: Inkinen, 2015. 


\section{Componentes básicos del capital intelectual (cI)}

Diversos estudiosos del tema coinciden en reconocer la composición básica del $\mathrm{Cr}$ en tres elementos: capital humano (сH), capital relacional (CR) y capital estructural (CE) u organizacional (Co) (Marzo y Scarpino, 2016; Aisenberg et al., 2015; Inkinen, 2015; Kianto, Ritala, Spender y Vanhala, 2014; Sydler, Haefliger y Pruksa, 2014; Berzkalne y Zelgalve, 2014; Dumay y Garanina, 2013; Rexhepi, Ibraimi y Veseli, 2013; Ienciu y Matiş, 2012; Kianto, Hurmelinna-Laukkanen y Ritala, 2010; Bueno et al., 2008; Mouritsen, 2006). El ch se compone de aquellos activos que se encuentran en los empleados, en su competencia, actitud y habilidad intelectual (Ienciu y Matiş, 2012; Engström et al., 2003), formación, habilidades, capacidades y características (Inkinen, 2015; Rexhepi et al., 2013), nivel de satisfacción, valores, motivación, creatividad, trabajo en equipo, resolución de problemas, habilidades de comunicación, liderazgo, y confianza (Dumay y Garanina, 2013; Rexhepi et al., 2013), remarcando que este capital no pertenece propiamente a la empresa (Sydler et al., 2014) sino a cada persona, lo que lo hace volátil (Edvinsson, 1997). El cH es considerado como el activo más importante del aprendizaje organizacional (Engström et al., 2003), ya que permite que los otros capitales sean formalizados (Aisenberg et al., 2015), además de que les brinda soporte (Inkinen, 2015), lo que significa que sin él, los otros pierden su valor.

El CR es visto como el valor y conocimiento obtenidos de las relaciones de las empresas con el exterior en todas sus dimensiones: clientes, proveedores, distribuidores, socios, comunidad local y otras entidades vinculadas como el gobierno (Inkinen, 2015; Sydler et al., 2014; Dumay y Garanina, 2013; Rexhepi et al., 2013). Se adquiere de la habilidad de la empresa para establecer relaciones positivas con sus stakeholders (Rexhepi et al., 2013) haciendo posible la actualización y mejoramiento de los otros capitales mediante la captación de información que obtiene de ellos (Kianto et al., 2010). Los conocimientos se convierten en insumos para desplegar la capacidad de innovación de productos, técnicas y procesos, así como la relación con actores del entorno (gobierno, medio ambiente, comunidad local) que les permite establecer alianzas formales e informales de colaboración y beneficio.

Así como el ch no pertenece a la empresa, sino que solo lo renta, el CR se encuentra parcialmente fuera de ella (Ienciu y Matiş, 2012; Engström et al., 2003), 
pues la relación puede estar al nivel de las personas o de las organizaciones. Cuando radica en las personas y dejan de colaborar en las empresas, este capital puede mudarse al menos de manera parcial junto con la persona, mientras que cuando radica en la organización permanecerá en ella con independencia de quien ocupe los puestos (Ienciu y Matiş, 2012).

El tercer componente básico del cr es el cE, compuesto del conocimiento que se encuentra arraigado en la empresa a través de los sistemas de información, documentos, bases de datos, descripción de procesos, planes, propiedad intelectual (Inkinen, 2015; Ienciu y Matiş, 2012; Kianto et al., 2010), estrategia de la organización, cultura organizacional, filosofía empresarial, marcas, logotipos (Sydler et al., 2014; Dumay y Garanina, 2013; Rexhepi et al., 2013; Ienciu y Matiş, 2012), sistemas, patentes, secretos industriales e innovación (Rexhepi et al., 2013), aunque también incluye el contexto en el que esos procesos toman lugar (Kianto et al., 2010), es decir, el ambiente laboral, entre otros. Se le considera el almacén de conocimientos de la empresa (Engström et al., 2003) perteneciente en su totalidad a ella cuando los empleados se han marchado (Roos, Roos, Dragonetti y Edvinsson, 2001).

La importancia del CE radica en ser el responsable de que la organización se mantenga en marcha (Aisenberg et al., 2015); se relaciona con el cH, pues la sistematización de la información, estandarización de los procesos y demás supone una independencia de las personas que ocupan los distintos puestos, ya que la empresa tiene en este capital los elementos necesarios para llevar a cabo sus tareas. De lo anterior se infiere que el cE es el de mayor valía en cuanto a la propiedad y aplicación que tiene como factor de apalancamiento (Edvinsson, 1997).

\section{Medición y reporte del cI}

Como consecuencia de la conciencia sobre la relevancia del cr en las empresas y su capacidad en la generación de valor, aparecieron estudios que se centraron en su medición a través de métodos cuantitativos, analizándolo en su conjunto o por cada una de sus partes; en su mayoría, han buscado atender las particularidades de cada sector o industria (Benavides, 2012). De los modelos surgidos para su medición e integración de reportes, Enríquez, Revah, Cruz y Romero 
(2007) diferencian aquellos que realizan una valoración financiera de los que se enfocan en el desempeño en las organizaciones, los que se limitan a reconocer su presencia y contribución en un nivel de apreciación, y aquellos que proponen indicadores para medirlo.

De los primeros se identifican el Human Resource Costing Accounting (HRCA) de Johansson (1996), el Value Added Intellectual Coeficient (varc) de Pulic Capital Intellectual (2000), el Intellectual Asset Valuation de Sulivan (2000), o el modelo de valor extracontable de Nevado y López (2002).

En los modelos correspondientes a la segunda categoría se hace una valoración del cr en función del desempeño; entre ellos están el Balance Scorecard de Kaplan y Norton (1992), el Holistic Value Approach de Roos, Roos, Dragonetti y Edvinsson (1997), o el Inclusive Valuation Methodology de Mc Pherson (2000). Berzkalne y Zelgalve (2014) señalan que estos modelos proponen medir la eficiencia con la que son empleados los elementos del cr en una organización.

En el tercer grupo se encuentran aquellos modelos que realizan solo una apreciación de su valor, como el Intellectual Capital Benchmarking System de Viedma (2001).

Por último, entre aquellos que únicamente observan indicadores para su medición se hallan el capital del conocimiento de Konrad Group (1989), el Skandia Navigator de Edvinsson y Malone (1997), y el Intellectual Capital Statement de Mouritsen (2001) (Enríquez, Revah, Cruz y Romero, 2007).

De manera similar, Nava (2010) agrupa los modelos de medición de acuerdo con su influencia en la organización. Los clasifica en aquellos que consideran que el ci tiene efectos unilaterales (principalmente económicos o en la productividad), multidimensionales (no solo resultados económicos sino también administrativos) e interdimensionales (aquellos cuyos efectos se notan en diversas áreas de las organizaciones, pero además con sinergias) (Nava, 2010).

De igual forma que sucedió con los componentes del cI, los modelos de medición que se crearon persiguieron fines concretos y particulares. En algunos casos, las mediciones pretendieron constituirse en guías generales de medición y reporte, como The Meritum Proyect para la Unión Europea, que tuvo lugar en Dinamarca de 1997 a 2002, donde participaron la Agencia de Industria y Comercio y posteriormente el Ministerio de Ciencia, Tecnología e Innovación, quienes construyeron y propusieron la Guía para la Declaración 
del CI, con la finalidad de involucrar al sector industrial del país en su reporte (Alcaniz, Gomez-Bezares y Roslender, 2011); así como Hong Kong, que involucró a más de 500 pequeñas y medianas empresas como estrategia para resaltar su importancia (Dumay y Garanina, 2013); sin embargo, algunos dejaron de lado su aplicación generalizada a sectores, industrias o países por presentar dificultades (Nava, 2010).

El caso de Dinamarca inició con 17 empresas consultoras e investigadoras, y con el tiempo llegó a involucrar alrededor de 100 empresas de diferentes sectores, tamaños y tipo de propiedad; no obstante, contrario a lo pretendido, Schaper (2016) encuentra en tal proyecto que muchas de las empresas abandonaron la práctica de declaración de su cr, por no encontrarle un beneficio en la organización, considerándolo solo como una moda o porque la dirección de las empresas estimó que no era un programa prioritario. Es decir, el mero reporte del stock de ci que cada empresa posee no les representó mayores beneficios como estrategia. Su tenencia, medición y reporte no constituyeron por sí mismos una ventaja competitiva.

El hecho de que las empresas hayan considerado a la medición y reporte de CI como programas de moda, sin beneficios verificables y, por lo tanto, programas no prioritarios, no implica que no los tenga, sino que de manera estática no los produce, y el foco de atención demanda centrarse en el empleo que de él se hace en la estrategia empresarial (incluida su medición), esto es, en conocer cuál es la dinámica del cI, cuáles son las relaciones de sus componentes, cómo se está empleando en las empresas para la creación de valor (Aisenberg et al., 2015) y con qué resultados para su gestión, dando lugar a una tercera etapa en su investigación (Guthrie, Ricceri y Dumay, 2012; Dumay y Garanina, 2013).

\section{Del cr estático al cr dinámico}

Ante los constantes cambios en los mercados y la falta de una aplicación rápida de la tenencia o stock de cr, las empresas se ven en dificultades para alcanzar una mayor competitividad (Eisenhardt y Martin, 2000). Dicho de otro modo, si no se emplean los componentes del cı pierden su valor y dejan de constituir una ventaja para las empresas (Kianto et al., 2010; Inkinen, 2015), ya que no son un fin en sí mismos, sino medios para conseguir otros fines. Por lo tanto, se necesita 
llevar a cabo acciones (capacidades empresariales) para crearlo, incrementarlo y gestionarlo para que genere valor, además de protegerlo de la imitación o de su apropiación por la competencia (Giuliani, 2015; Kianto et al., 2010).

A finales del siglo pasado, Teece hacía referencia al término capacidades dinámicas como "la capacidad de la empresa para integrar, construir y reconfigurar tanto los recursos como las competencias funcionales" (Teece et al., 1997, p. 516). Otros autores que coinciden en la importancia del dinamismo en el cr de las empresas son Vivas-López (2013, p. 129), quien menciona la creación de "rutinas organizacionales de carácter estratégico a través de las cuales las empresas obtienen nuevas configuraciones de recursos cuando los mercados emergen, chocan, se dividen o evolucionan”, y Eisenhardt y Martin (2000), quienes sostienen que estas capacidades son aquellos procesos que se gestionan para alterar los recursos y convertirlos en fuentes de ventaja competitiva en esos mercados que cambian de manera constante.

Cuando las capacidades dinámicas se enfocan al cr, se busca reconfigurar los recursos y competencias funcionales, tomando como base el aprendizaje organizacional y el conocimiento (Marzo y Scarpino, 2016), aprovechando estratégicamente los activos intangibles para hacer palpable su contribución en la creación de valor (Aisenberg et al., 2015). Las capacidades dinámicas y la innovación son las responsables en el desempeño de las empresas, y su competitividad es el resultado del efecto combinado del CH, CR y CE, a través de su gestión deliberada y sistemática. El ci debe ser incitado para producir los efectos que de él se esperan (Giuliani, 2015; Inkinen, 2015).

Con base en ello, Kianto, Hurmelinna-Laukkanen y Ritala (2010) dividen los enfoques del cr en estáticos y dinámicos, postura que ha sido sostenida por otros autores (Marzo y Scarpino, 2016; Giuliani, 2015). Esa división consiste en que el cr estático pretende identificar el listado de activos que las empresas poseen y tiene por objetivo su evaluación sin reparar en sus relaciones, sino identificarlos individualmente en un contexto caracterizado por vínculos débiles entre los tres componentes (Marzo y Scarpino, 2016), en tanto, el cr dinámico busca dirigirlo (Bueno et al., 2008); las relaciones entre los componentes están fuertemente estructuradas y tienen la capacidad de marcar diferencias en las empresas. Es desde esas interrelaciones del CH, CE y CR que se puede llegar a producir una teoría dinámica del ci (Mouritsen, 2006) refiriéndose a las actividades 
empleadas para la creación, gestión y coordinación de esos activos, actividades que también han sido denominadas por algunos como Know-ledge Management (Kianto et al., 2014).

Para que el cr tenga resultados en las empresas es necesaria la interacción de sus tres componentes, pues "el cH se requiere para establecer el cE y este es requerido para construir el CR con el ambiente” (Inkinen, 2015, p. 529).

Mouritsen (2006) emplea los términos "performativo" y "ostensivo" para referirse a la diferenciación de las dos perspectivas, de las cuales el primero alude a la forma en que se combina y gestiona estratégicamente para el logro de los objetivos, además de remarcar en su enfoque "lo que el cr hace", mientras que el segundo está relacionado con el enfoque estático: "lo que el cr es” (Giuliani, 2015).

Desde el enfoque dinámico, el cr se concibe a partir de cómo es utilizado en las empresas en sus actividades diarias, cómo se aplica para crear valor o para convertirlo en ventajas competitivas (Giuliani, 2015), cómo se renueva y de qué modo se protege de la imitación (Kianto et al., 2010), conscientes del papel que tiene para su estructura, procesos y creación de valor (Marzo y Scarpino, 2016); aunque no necesariamente con ese nombre, pues las empresas pueden no identificar que realizan prácticas que involucran el cı y lo emplean estratégicamente, pero sí con la certeza de sus resultados y de manera sistemática como parte de su estrategia.

Para Marzo y Scarpino (2016), la división del ci en CH, CE y CR (enfoque estático) queda solo en la perspectiva de inicio, pues ante el dinamismo no es posible analizarlos por separado, cuestión coincidente con Giuliani (2015), quien apunta la dificultad de clasificar las relaciones en su dinamismo, puesto que resultan en procesos complejos.

Otra diferencia entre los dos enfoques es que la tenencia de cr en las empresas provoca la heterogeneidad entre ellas, o sea, son diferentes entre sí cuando se analiza su stock; pero desde el enfoque dinámico, las prácticas pueden tener cierta similitud, la gestión del cr es más homogéneo, los procedimientos y las rutinas empleados pueden ser equiparables entre las empresas, su empleo tiende a ser similar en un mismo sector industrial (Eisenhardt y Martin, 2000). Esto se debe a que buscan objetivos semejantes: aprovechar, hacer, crecer, emplear estratégicamente el cr para la creación de ventajas competitivas, sin que ello 
represente patrones que se implementen idénticamente en todas las empresas, sino que el dinamismo del mercado, su entorno y características particulares determinarán su perspectiva (Eisenhardt y Martin, 2000).

Kianto et al. (2010) agrupan ese dinamismo en creación, gestión y protección. Entre aquellas que lo crean está la implementación de capacidades organizacionales y procesos que facilitan el aprendizaje y la innovación en su interior. Es decir, las actividades que de manera organizada y sistemática favorecen que sus miembros aprendan, obtengan y desarrollen conocimientos relativos a procesos, productos, servicios, clientes, o que conviertan la experiencia, habilidades y técnicas en referentes para la empresa, así como aquellas que fomentan la innovación en esos mismos u otros rubros. En las empresas de servicios, la flexibilidad y creatividad se traducen en incremento de valor para los clientes.

Algunas de estas prácticas consisten en la estimulación del aprendizaje a partir de prueba-error como fuente de nuevos conocimientos o en desafíos para buscar nuevos métodos y prácticas, colaboración, observación y aprendizaje entre diferentes departamentos y la retroalimentación conjunta, junto con acciones que miden si el cr se ha incrementado, mantenido o disminuido (Kianto et al., 2010).

Las actividades de gestión se refieren a su aprovechamiento para obtener su máximo potencial. Las empresas definen en la estrategia cómo se empleará el Cr y qué es lo que se espera obtener de él en la generación de valor agregado o ventaja competitiva (Rexhepi et al., 2013).

En este rubro, algunas prácticas consisten primero en la difusión y comprensión de la estrategia, seguidas del desarrollo de habilidades y conocimiento organizacional, proyectos y prácticas destinados a incrementar las capacidades, habilidades y conocimientos de los empleados, que redunde en el desarrollo de nuevos procesos, productos y servicios, o en su mejoramiento.

En tercer lugar están las actividades desarrolladas para proteger el cr de la imitación o de que sus activos intangibles sean adquiridos por la competencia, pues aunque el benchmarking es una práctica usual, la empresa que de manera efectiva logra obtener una ventaja competitiva en el sector buscará protegerla y se valdrá de acciones para resguardarla, de forma que aproveche para sí los mayores beneficios antes de que sea imitado. Estas actividades incluyen el registro de derechos de propiedad intelectual y la secrecía, así como la 
protección legal relativa a los empleados, prácticas de gestión de recursos humanos que pretendan su permanencia, métodos de ocultamiento técnico, acuerdos de confidencialidad y exclusividad, complejidad en el proceso, producto o servicio, plazos de entrega e inclusive acciones de inutilización de recursos en ambientes distintos, entre otras. Particularmente en el sector servicios las actividades de protección del cr tienden a ser más débiles dadas las características propias de intangibilidad (Kianto et al., 2010).

Marzo y Scarpino (2016) refuerzan esta visión al señalar que la estandarización de procesos (como creación de cI) permite en la empresa que los requerimientos para la contratación del personal temporal se limiten a conocimientos genéricos y que el aprendizaje sea continuo en la realización de las actividades cotidianas. Por el contrario, el personal fijo y con funciones clave para la empresa ha de desarrollarse en habilidades avanzadas de negocio y habilidades de valuación, como perspectiva de gestión del capital humano.

En el caso empírico estudiado por Marzo y Scarpino (2016), el desarrollo de habilidades particulares en el personal tiene consecuencias hacia la gestión y creación de valor al cliente. Proveer al personal de habilidades de relación con el cliente, el conocimiento de la estrategia empresarial, sumado al conocimiento genérico que tiene de su sector, permite que en la interacción, las peticiones y requerimientos se materialicen en el desarrollo de nuevos productos, a partir de ideas ambiguas o peticiones especiales, representando una nueva ventaja para la empresa respecto a la competencia. En este caso se resalta el rol que tienen los clientes en el desarrollo de nuevos conocimientos, la colaboración con proveedores y la competencia, la compartición de información principalmente técnica, resultando en mejoras operacionales y disminución de costos.

Como puede apreciarse, el ci dinámico consiste en la implementación y uso del ci en la estrategia organizacional, una vez que se ha reconocido como generador de ventajas competitivas y valor agregado.

\section{Hotelería y ci}

De acuerdo con la Organización Mundial del Turismo (omT, 2015), la llegada de turistas internacionales (turistas entre diferentes naciones) ha mostrado un crecimiento casi sin interrupciones, contribuyendo a la consolidación de esta 
actividad como generadora de divisas, empleo y dinamismo económico para los países receptores (véase cuadro 2).

CUADRo 2. Llegada de turistas internacionales

\begin{tabular}{lcccccc}
$\quad$ Año & 1950 & 1980 & 1990 & 2000 & 2011 & $2015^{*}$ \\
$\begin{array}{l}\text { Millones de } \\
\text { turistas }\end{array}$ & 25 & 277 & 435 & 675 & 940 & 810 \\
\hline
\end{tabular}

* Hasta agosto de 2015.

Fuente: (www2.unwto.org)

Para el caso de México la situación es similar: en 2013 ingresaron al país 24.2 millones de turistas, los cuales representaron casi 14 millones de dólares en divisas (tercera fuente generadora para la nación) y el mayor crecimiento en el rubro comparado con los años anteriores, contribuyendo con $8.4 \%$ del producto interno bruto (Presidencia de la República, 2013) y la generación de más de 2.2 millones de empleos (Secretaría de Turismo, 2013, p. 23); para 2014 se alcanzaron los 29 millones de turistas (World Tourism Organization.2015).

La industria turística se relaciona mayormente con la transportación aérea, el hospedaje y la alimentación, juntas suman más de 43000 unidades económicas del sector, sobresaliendo la industria hotelera con una oferta de poco más de 17300 establecimientos en el nivel nacional (García, 2013). Por ello, la ocupación hotelera se considera como un parámetro clave del sector, representativo de su acontecer, la cual siguiendo la tendencia alcista presenta una ocupación de 60.7 \% generalizado nacional (Secretaría de Turismo, 2015), además de que de enero a julio de 2015 la llegada de turistas nacionales a cuartos de hotel superó los 30.1 millones de personas, correspondiendo a $75.4 \%$ del total, mientras que el $24.6 \%$ restante concernió a turistas extranjeros (Secretaría de Turismo, 2015, p. 9).

La industria hotelera, debido a las características de sus servicios y la diferencia con otras industrias, como la relación directa entre el prestador del servicio y el cliente durante el proceso, la intangibilidad, la no acumulación, producción y consumo en el mismo instante, la tecnología como soporte de servicios y procesos (Mejía, 2015), la imposibilidad de probarse antes de su disfrute (Kianto et al., 2010; Lillo et al., 2007), el ambiente impredecible y complejo en el que 
operan (Manosalvas y Manosalvas, 2014), entre otras, hace más notoria la importancia que tiene el cr en este tipo de empresas confiriéndole particular atención para su estudio (Bravo et al., 2014; Gonçalves, Sass, Rastrollo y Savi, 2014; García, 2013; Kianto et al., 2010; Gazzera y Vargas, 2008; Engström et al., 2003), al detectarse que estas cualidades revelan una alta dependencia (Kianto et al., 2010).

De ahí que se destaque que el ch y el cE tienen un papel preponderante en la hotelería, por una parte, por la heterogeneidad de los mercados turísticos, y la personalización y calidad de los servicios que demandan; pero también por la incorporación de la tecnología en sus procesos y estructuras organizacionales, lo que le adiciona complejidad a las cualidades demandadas de esos capitales y la forma en que pueden ser aprovechados (Lillo et al., 2007).

En ambos capitales la hotelería encuentra desafíos para elevar su competitividad, pues, por un lado, la alta rotación del personal operativo provoca que los activos que radican en él se pierdan (Gonçalves et al., 2014), mientras que en hoteles que no pertenecen a cadenas hoteleras, la carencia de soportes estructurales limitan su organización, crecimiento y desarrollo. En este sentido, se remarca la necesidad de la innovación de servicios, mejoras en la forma de atender a los clientes y gestión de los procesos, los cuales tienen que ver con el manejo de los activos intangibles y su empleo de manera estratégica (Lillo et al., 2007; Manosalvas y Manosalvas, 2014).

Por lo tanto, si se hace notar que el ci dinámico tiende a homogeneizar la gestión, los procedimientos y las rutinas (Eisenhardt y Martin, 2000), pueden encontrarse prácticas comunes para el sector hotelero atendiendo a sus particularidades para la creación de ventajas competitivas. Por ejemplo, si el cH en la hotelería resulta clave para su óptimo aprovechamiento como activo en la producción de servicios, las prácticas de protección y desarrollo estratégico basados en él tenderían a ser clave para toda la industria hotelera, con independencia de las particularidades de cada hotel.

Del mismo modo, si la relación y comunicación con el cliente y stakeholders se consideran estratégicas para la obtención de información en el diseño de nuevos productos y procesos, lo será para todo el ramo, así como su gestión, correspondiendo a cada empresa el diseño de las formas para su obtención, codificación y desarrollo. 
Consecuentemente, se afirma que es factible y conveniente proponer un modelo de gestión de ci dinámico para la hotelería, a partir de la identificación de aquellas prácticas que pueden encontrarse en los hoteles y que son empleadas de manera sistemática en la gestión, para su aprovechamiento con la finalidad de mejorar su desempeño y elevar su competitividad.

\section{Fundamento metodológico}

La importancia de la actividad turística queda manifiesta por su contribución al producto interno bruto y la creación de empleos. Por sus características, las empresas hoteleras enfrentan problemas que limitan sus capacidades de aprendizaje, de innovación y para reorganizarse, debido a la falta de sistematización de la información generada a partir de sus relaciones, a la demanda de implementación de tecnologías de la información que hagan eficientes sus procedimientos y a la alta rotación de personal que ocasiona que se pierdan conocimientos y experiencia, entre otros factores valiosos, repercutiendo en sus niveles de competitividad, por lo que se requieren estudios que contribuyan a resolver esta problemática.

La pregunta que fundamenta esta investigación es: ¿qué activos y prácticas del Cr se pueden implementar en las empresas del sector hotelero para transitar del cI estático al cr dinámico e incrementar sus ventajas competitivas? De aquí que el objetivo es la identificación de activos intangibles claves de la hotelería y los procesos estratégicos que posibiliten su tránsito del cr estático al dinámico.

Esta investigación corresponde a un estudio exploratorio, basado en una revisión de literatura en la que se tomaron como base 47 artículos de diversas revistas científicas entre las que destacan el Journal of Intellectual Capital (JIC), Tourism \& Management Studies, International Review of Business Research y British Accounting Review. En primera instancia, los criterios de búsqueda y selección fueron las palabras clave y el contenido del abstract, enfocándose en aspectos del cr como componentes básicos, su medición y reporte, tránsito del cr estático al dinámico, y su relación con la hotelería. La información obtenida en los artículos se complementó con aquella proveniente de bibliografía especializada y páginas oficiales de organismos vinculados al turismo y la hotelería. En total se tomaron 38 referencias, de las cuales sobresalen los estados del arte 
realizados por Zambon (2016), Aisenberg, Durst, Zaniboni y Selig (2015), Dumay y Garanina (2013), Guthrie, Ricceri y Dumay (2012), e Ienciu y Matiş (2012), quienes constituyeron el sustento básico para esta fundamentación teórica.

Tras el análisis de la información e identificación de los elementos más relevantes de cada componente, se procedió al desarrollo de la propuesta para transitar del cr estático al dinámico en la hotelería, partiendo del reconocimiento de la importancia del cr en sus dimensiones humana, estructural y relacional, teniendo en cuenta las características particulares de la industria hotelera y la función del cr en la prestación de sus servicios y la búsqueda de mayores niveles de competitividad.

\section{Hacia un cr dinámico en la hotelería}

A partir de la investigación desarrollada, se presenta la propuesta del proceso de transición del cr estático al dinámico para el sector hotelero (véase figura 1).

Para lograr la transición del cr estático al ci dinámico es necesario atender los aspectos señalados en los cuadros 3, 4 y 5 .

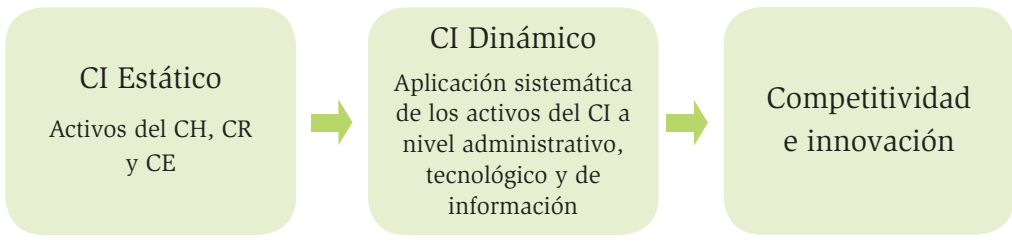

Fuente: Elaboración propia.

Figura 1. Proceso de transición del CI Estático al CI Dinámico en el sector hotelero

Además de ser visto a partir de la operación particular que realiza, es necesario considerar las capacidades del cн en función del cг para aprovecharlo, emplearlo y desarrollarlo. Si se le selecciona y gestiona de esta forma, no solo se incrementará el cr empresarial, sino que aumentará la posibilidad de crearlo, gestionarlo y producir a partir de él. De llevarse a cabo, los hoteles requerirán medidas para protegerlo, pues si los resultados son palpables se esforzarán en su retención y desarrollo, antes que exponerse a perder conocimientos y cualidades debido a la rotación (Gonçalves et al., 2014). 
CuAdro 3. Transición del CI Estático al CI Dinámico basado en el capital humano

CI Estático (activos)

Habilidades, capacidades, valores, experiencia

Experiencia
CI Dinámico (procesos)
Resultados potenciales

Creación e incremento del cI

Procesos de selección de personal en función de la estrategia hotelera CI

Mecanismo de acopio de experiencia del personal para su uso estratégico

Habilidades, conoci-

Mecanismos que incentiven miento, experiencia la innovación en productos

Iniciativa, experiencia y habilidades

Mecanismos que incentiven en el personal la innovación en procesos

Iniciativa, experiencia y habilidades

Mecanismos que incentiven la innovación de la estructura organizacional

Experiencia, habilida- Participación general para el des, trabajo en equipo rediseño de la estructura organizacional

Conocimientos, habilidades, experiencia

Benchmarking que involucre a todo el personal (operativo y administrativo)

Habilidades, conocimientos, destrezas

Habilidades, Mecanismos tendientes a la retención del personal

Análisis de los recursos y conocimintos, valores

capacidades del personal para el diseño de planes de carrera

Experiencia, habilida- Fomento de la participación del des, conocimiento personal en diferentes áreas para enriquecer su conocimiento en la cadena de valor

Experiencia, conocimientos, habilidades

Documentación de los errores o fallas en los procesos de atención al huésped como oportunidad de desarrollo

Experiencia, Fomento de la participación de conocimientos, todo el personal en los seminarios, reuniones, conferencias y servicios

Nuevos procesos, productos y servicios

Innovación en procesos

Reestructuración

organizacional

Involucramiento y compromiso empresarial en ambas direcciones

Incremento de las capacidades de análisis para el mejoramiento organizacional

Protección y retención del cH

Protección y retención del cH, así como incremento de los activos intangibles en ese capital

Adquisición de mayores conocimientos e identificación de la labor como parte de un sistema

Sistematización de la búsqueda de áreas de mejora para todos los capitales

Incremento de las capacidades del ch para su aprovechamiento en la organización
Nuevos procesos, productos 


\section{Cuadro 4. Transición del CI Estático al CI Dinámico basado en el capital relacional}

\begin{tabular}{|c|c|c|}
\hline $\begin{array}{c}\text { CI Estático } \\
\text { (activos) }\end{array}$ & $\begin{array}{l}\text { CI Dinámico } \\
\text { (procesos) }\end{array}$ & Resultados potenciales \\
\hline Relación con clientes & $\begin{array}{l}\text { Registro y análisis de las peti- } \\
\text { ciones de las huéspedes }\end{array}$ & $\begin{array}{l}\text { Nuevos productos y mejora } \\
\text { de procesos }\end{array}$ \\
\hline Relación con clientes & $\begin{array}{l}\text { Mecanismo tendientes a } \\
\text { obtener información de los } \\
\text { huéspedes }\end{array}$ & $\begin{array}{l}\text { Retroalimentación, } \\
\text { información para mejora de } \\
\text { productos y servicios }\end{array}$ \\
\hline $\begin{array}{l}\text { Relación con clientes, } \\
\text { proveedores, gobierno, } \\
\text { competencia, entorno }\end{array}$ & $\begin{array}{l}\text { Establecimiento de protocolo } \\
\text { para el desarrollo de relacio- } \\
\text { nes como fuente de informa- } \\
\text { ción, conocimiento y de valor }\end{array}$ & $\begin{array}{l}\text { Obtención de información } \\
\text { clave, alianzas, cooperación } \\
\text { y aprendizaje organizacional }\end{array}$ \\
\hline $\begin{array}{l}\text { Relación con } \\
\text { stakeholders }\end{array}$ & $\begin{array}{l}\text { Documentación de las con- } \\
\text { diciones y propósitos de las } \\
\text { relaciones con stakeholders }\end{array}$ & $\begin{array}{l}\text { Apropiación del valor de } \\
\text { las relaciones con los } \\
\text { stakeholders }\end{array}$ \\
\hline
\end{tabular}

Fuente: Elaboración propia.

\section{Cuadro 5. Transición del CI Estático al CI Dinámico basado en el capital estructural}

\begin{tabular}{|c|c|c|}
\hline $\begin{array}{l}\text { CI Estático } \\
\text { (activos) }\end{array}$ & $\begin{array}{l}\text { CI Dinámico } \\
\text { (procesos) }\end{array}$ & Resultados potenciales \\
\hline $\begin{array}{l}\text { Procesos, sistemas, } \\
\text { rutinas }\end{array}$ & $\begin{array}{l}\text { Revisión, documentación y } \\
\text { revisión de procesos, rutinas } \\
\text { y sistemas }\end{array}$ & $\begin{array}{l}\text { Actualización de procedi- } \\
\text { mientos de producción y } \\
\text { servicio }\end{array}$ \\
\hline $\begin{array}{l}\text { Procesos, sistemas, } \\
\text { bases de datos }\end{array}$ & $\begin{array}{l}\text { Incorporación de sistemas y } \\
\text { procedimientos basados en } \\
\text { las TIC }\end{array}$ & $\begin{array}{l}\text { Mejoramiento de procesos y } \\
\text { de la atención a huéspedes. } \\
\text { Incremento del cE }\end{array}$ \\
\hline $\begin{array}{l}\text { Valor de la marca, } \\
\text { imagen corporativa }\end{array}$ & $\begin{array}{l}\text { Fortalecimiento y en su caso } \\
\text { rediseño de la marca con } \\
\text { orientación al servicio y la } \\
\text { hospitalidad }\end{array}$ & $\begin{array}{l}\text { Proyección del valor de la } \\
\text { marca asociada a característi- } \\
\text { cas del servicio }\end{array}$ \\
\hline $\begin{array}{l}\text { Filosofía empresarial } \\
\text { centrada en el cI }\end{array}$ & $\begin{array}{l}\text { Prácticas que refuerzan el } \\
\text { valor del conocimiento y la } \\
\text { información para el logro de } \\
\text { las metas empresariales }\end{array}$ & $\begin{array}{l}\text { Comprensión sobre la impor- } \\
\text { tancia del cI para el desarrollc } \\
\text { empresarial }\end{array}$ \\
\hline $\begin{array}{l}\text { Filosofía empresarial } \\
\text { centrada en el cI }\end{array}$ & $\begin{array}{l}\text { Difusión y comprensión } \\
\text { de la estrategia del cI }\end{array}$ & $\begin{array}{l}\text { Comprensión de la estrategia } \\
\text { centrada en el valor del cI }\end{array}$ \\
\hline Todo el stock del cI & Medición del cI & $\begin{array}{l}\text { Evaluación de los resultados } \\
\text { obtenidos de la gestión del cI }\end{array}$ \\
\hline
\end{tabular}


La aplicación de medidas enfocadas a la sanción del personal por fallas en el servicio en la hotelería limita su capacidad de exploración de nuevas formas para su mejoramiento, así como la confinación del $\mathrm{cH}$ a un solo campo de acción por no tener una visión general del producto ofrecido.

La innovación de productos en la hotelería puede provenir de diferentes fuentes, por lo que, de manera general, la estimulación para su generación debe comprender la participación de todas las áreas y de forma sistemática.

Por ejemplo, las peticiones especiales de los huéspedes sirven de fuente de información para la innovación de servicios y procedimientos, no solo para aquellos que están en contacto con el cliente, sino para toda la cadena productiva de la organización, por lo que su recopilación y difusión requiere ser total.

Sin embargo, no es la única fuente; toda interacción con los stakeholders puede aportarla, haciendo necesarios protocolos de gestión de relaciones y documentación de su valor obtenido para la gestión.

Si el CE es pobre en procedimientos y sistemas para llevar a cabo sus acciones, no se podrá aprovechar el potencial del ci (Engström et al., 2003).

En esto hay que diferenciar a la tecnología del cE, pues a pesar de que están muy relacionados, no todo se encuentra contenido en ella. Así, aunque los recursos financieros y tecnológicos sean limitados, el cE puede desarrollarse y gestionarse para aportar valor, pues lo que de él se demanda es el soporte necesario y la sistematización, para que el ch se mantenga en operación y cada vez en mejores condiciones, garantizando un nivel de atención acorde con el objetivo.

Por ello los hoteles deben priorizar que todo su personal comprenda el valor que tienen estos activos y su empleo, de modo que la revisión constante y generalizada de procesos, sistemas y formas de organización forman parte de las claves para la mejora del desempeño organizacional.

Asimismo, el personal debe comprender cuál es la estrategia seguida por la empresa, pues se considera que solo entre 5 y $8 \%$ del total de los empleados la entienden, lo que representa una limitante y un reto que enfrentan los gerentes (Rexhepi et al., 2013, p. 48).

Finalmente, la medición del ci por sí mismo no crea valor, pero si ha sido empleado estratégicamente buscando su creación, gestión y protección, es nece106 sario su medición como mecanismo de evaluación de los resultados de impacto de la estrategia. 


\section{Conclusiones}

Las condiciones del mercado resaltan cada vez más la importancia del ci como fuente de creación de valor y consecuentemente de ventajas competitivas para las empresas, y en la hotelería presenta características particulares como la intangibilidad, producción y consumo en el mismo instante e interacción con el huésped, además de la interrelación que tiene con la implementación de tecnologías.

Una aportación significativa de la propuesta tiene que ver con el beneficio que puede obtenerse de la gestión de los elementos del cr, primero considerándolo como generador de valor y, después, empleándolo en los procesos.

Para que las prácticas de ci se conviertan en estrategia enfocada en elevar la competitividad hotelera, es necesaria su sistematización en los procesos administrativos, la implementación de tecnología y gestión de la información. Se requiere el aprovechamiento metódico de los conocimientos y capacidades del ch, así como su valoración en función de la estrategia de ci desde el momento de su reclutamiento y extenderlo hacia su permanencia.

En ese tenor, son los requerimientos para que la información generada a partir del cr pueda capitalizarse hacia la innovación y el mejoramiento de procesos, servicios y productos.

La implementación de sistemas y la innovación de modelos de organización y de tecnología en la hotelería contribuyen a consolidar el cE, por lo que la inversión que se haga en ello favorecerá la sinergia necesaria con los otros dos capitales para elevar la competitividad del sector.

Es menester transitar del cr estático al dinámico a través de su aplicación estratégica y llevarlo a la hotelería, como modelo de gestión para incrementar sus ventajas competitivas, en un mercado global y con altos niveles de competencia. Para lograr esta transición es fundamental pasar de la disponibilidad de activos intangibles a acciones reales y tangibles enmarcadas por estrategias y procesos establecidos para la interacción de los componentes del CH, CR y CE.

\section{Alcances, limitaciones y trabajo futuro}

Entre los alcances de la presente investigación destaca el esfuerzo realizado para profundizar en el estudio del cr vinculándolo a la industria hotelera para 
impulsar su competitividad; la revisión de literatura incluyó investigaciones del cr plasmadas en 47 artículos científicos de prestigiadas revistas internacionales especializadas en capital intelectual, turismo y gestión organizacional. Los estudios revisados incluyeron referentes teóricos y empíricos, aunque no propiamente del enfoque dinámico en la hotelería, lo que constituye una limitación.

La propuesta del estudio plantea la posibilidad de llevar a cabo estudios empíricos enfocados en el impacto del cr dinámico en la hotelería, que permitan validar la aplicación del proceso de transición propuesto y constatar cómo es la relación entre este y la competitividad del sector. Por otra parte, su aplicación puede extenderse a empresas de otro sector.

\section{Fuentes consultadas}

Aisenberg, H., Durst, S., Zaniboni, M. y Selig, P. (2015). Intellectual capital dimensions: State of the art in 2014. Journal of Intellectual Capital, 16(1), 58-100. Recuperado de http://doi.org/10.1108/JFM-03-2013-0017

Alcaniz, L., Gomez-Bezares, F. y Roslender, R. (2011). Theoretical perspectives on intellectual capital: A backward look and a proposal for going forward. Accounting Forum, 35(2), 104-117. Recuperado de http://doi. org/10.1016/j.accfor.2011.03.004

Benavides, L. E. (2012). Medición, valoración y determinación del impacto del Capital Intelectual en la generación de valor en la empresa. Tendencias, Revista de la Facultad de Ciencias Económicas y Administrativas, XIII(1), 100-115.

Berzkalne, I. y Zelgalve, E. (2014). Intellectual capital and company value. Procedia-Social and Behavioral Sciences, 110, 887-896. Recuperado de http://doi.org/10.1016/j.sbspro.2013.12.934

Bravo, J., Andrade, M. y Delgado, L. (2014). Retos de la inteligencia analítica como fuente de innovación en el sector hotelero: Revisión teórica. International Review of Business Research Papers, 10(3), 121-133.

Bueno, E., Salmador, P. y Merino, C. (2008). Génesis, concepto y desarrollo del capital intelectual en la economía del conocimiento: Una reflexión sobre el Modelo Intellectus y sus aplicaciones. Estudios de Economía Aplicada, 26(2), 43-63. Recuperado de http://doi.org/http://www. revista-eea.net 
Dumay, J. y Garanina, T. (2013). Intellectual capital research: A critical examination of the third stage. Journal of Intellectual Capital, 14(1), 10-25. Recuperado de http://doi.org/10.1108/14691931311288995

Edvinsson, L. (1997). Developing intellectual capital at Skandia. Long Range Planning, 30(3), 366-373. Recuperado de http://doi.org/10.1016/ S0024-6301(97)90248-X

Eisenhardt, K. y Martin, J. (2000). Dynamic capabilities: What are they? Strategic Management Journal, 21, 1105-1121.

Engström, T., Westnes, P. y Westnes, S. (2003). Evaluating intellectual capital in the hotel industry. Journal of Intellectual Capital, 4(3), 287-303. Recuperado de http://doi.org/10.1108/14691930310487761

Enríquez, S., Revah, B., Cruz, Y. y Romero, N. (2007). Valuación y reconocimiento de activos intangibles. Un enfoque estratégico. México: Talleres Gráficos.

García, S. (2013). Importancia estratégica de los activos intangibles en la industria hotelera. Hospitalidad-Esdai, enero-junio, 7-26.

Gazzera, A. y Vargas, E. E. (2008). El valor de los intangibles en las empresas prestadoras de servicios turísticos. Caso hotelería en Toluca, México. Gestión Turística, 9-26. doi:10.4206/gest.tur.2009.nesp-02

Giuliani, M. (2015). Intellectual captal dyamics: Seeing them "in practice" through a temporal lens. VINE, 45(1), 46-66.

Gonçalves, J., Sass, C., Rastrollo, M. y Savi, T. (2014). La gestión del conocimiento en cadenas hoteleras: Una revisión. Tourism \& Management Studies, 10(2), 146-154.

Guthrie, J., Ricceri, F. y Dumay, J. (2012). Reflections and projections: A decade of intellectual capital accounting research. British Accounting Review, 44(2), 68-82. Recuperado de http://doi.org/10.1016/j.bar.2012.03.004

Ienciu, N. y Matiş, D. (2012). The analysis of intellectual capital evolution: A perspective on research. Journal of Human Resource Costing \& Accounting, 16(4), 341-366. Recuperado de http://doi. org/10.1108/14013381211318346

Inkinen, H. (2015). Review of empirical research on intellectual capital and firm performance. Journal of Intellectual Capital, 16(3), 518-565. Recuperado de http://doi.org/http://dx.doi.org/10.1108/02683940010305270 
Kianto, A., Hurmelinna-Laukkanen, P. y Ritala, P. (2010). Intellectual capital in service- and product-oriented companies. Journal of Intellectual Capital, 11(3),305-325. Recuperadodehttp://doi.org/10.1108/14691931011064563

Kianto, A., Ritala, P., Spender, J.-C. y Vanhala, M. (2014). The interaction of intellectual capital assets and knowledge management practices in organizational value creation. Journal of Intellectual Capital, 15(3), 362375. Recuperado de http://doi.org/10.1108/JIC-05-2014-0059

Lillo, A., Ramón, A. y Sevilla, M. (2007). El capital humano como factor estratégico para la competitividad del sector turístico. Cuadernos de Turismo, 19, 47-69. Recuperado de http://dialnet.unirioja.es/descarga/ articulo/2316405.pdf

López, V. y Nevado, D. (2006). Gestione y controle el valor integral de su empresa. Madrid: Díaz de Santos.

López, V. y Nevado, D. (2016). Modelo de control estratégico desde la perspectiva del valor de los intangibles. Método y aplicación. Revista Innovar, 26(59), 9-20. Recuperado de http://doi.org/10.15446/innovar. v26n59.54319.CITACI

Manosalvas, C. y Manosalvas, L. (2014). Diagnóstico de los factores que influyen en la competitividad de las empresas de alojamiento. Global Conference on Business and Finance Proceedings, 9(2), 1661-1669.

Marzo, G. y Scarpino, E. (2016). Exploring intellectual capital management in SMEs. An in-depth Italian case study. Journal of Intellectual Capital, $17(1), 1-20$.

Mejía, M. (2015). Nuevas tecnologías para el desarrollo de la industria turística en Guanajuato, México. Rotur, Revista de Ocio y Turismo, 9, 35-43.

Mercado, P. Nava, R. M. y Demuner, M. R. (2012). Presencia de los intangibles y el capital intelectual en la competitividad país-empresa. En P. Mercado y A. D. Cernas (eds.), El capital intelectual en las organizaciones (pp. 17-43). México: Universidad Autónoma del Estado de México.

Mouritsen, J. (2006). Problematisingintellectual capital research:Ostensiveversus performative Ic. Accounting, Auditing \& Accountability Journal, 19(6), 820-841. Recuperado de http://doi.org/10.1108/09513570610709881

Nava, R. (2010). Modelo de capital intelectual para la UAEMex (2009) en el marco de la competitividad institucional. Toluca: Universidad Autónoma del Estado de México. 
Presidencia de la República (2013). Plan Nacional de Desarrollo 2013-2018. Recuperado de http://www.dof.gob.mx/nota_detalle.php?codigo $=52994$ $65 \&$ fecha $=20 / 05 / 2013$

Rexhepi, G., Ibraimi, S. y Veseli, N. (2013). Role of intellectual capital in creating enterprise strategy. Procedia-Social and Behavioral Sciences, 75, 44-51. Recuperado de http://doi.org/10.1016/j.sbspro.2013.04.006

Roos, J., Roos, G., Dragonetti, N. y Edvinsson, L. (2001). Capital intelectual. El valor intangible de la empresa. Barcelona: Paidós Empresa.

Saavedra, M. L. (2012). Una propuesta para la determinación de la competitividad en la pyme latinoamericana. Pensamiento \& Gestión, 33(50), 93-124.

Schaper, S. (2016). Contemplating the usefulness of intellectual capital reporting. Journal of Intellectual Capital, 17(1), 52-82.

Secretaría de Turismo. (2013). Resultados de la Actividad Turística 2013. México: Autor.

Secretaría de Turismo. (2015). Fondo Sectorial para la Investigación, el Desarrollo y la Innovación Tecnológica en Turismo. Recuperado en febrero 17 de 2016.

Secretaría de Turismo. (2015). Fondo Sectorial para la Investigación, el Desarrollo y la Innovación Tecnológica en Turismo. Recuperado de http:// www.conacyt.mx/index.php/el-conacyt/convocatorias-y-resultadosconacyt/convocatorias-fondos-sectoriales-constituidos/convocatoriasectur-conacyt-1/convocatoria-abierta-sectur/8975-demandas-del-sector-convocatoria-2015-01/file en febrero 17 de 2016.

Sydler, R., Haefliger, S. y Pruksa, R. (2014). Measuring intellectual capital with financial figures: Can we predict firm profitability? European Management Journal, 32, 244-259. Recuperado de http://doi.org/10.1016/j. emj.2013.01.008.

Teece, D., Pisano, G., y Shuen, A. (1997). Dynamic capabilities and strategic management. Strategic Management Journal, 18(7), 509-533.

Vivas-López, S. (2013). Implicaciones de las capacidades dinámicas para la competitividad y la innovación en el siglo XXI. Cuadernos de Administración, 26(47), 119-139.

World Economic Forum (2013). The Human Capital Report, Cologny/Geneva, Switzerland: World Economic Forum. Recuperado de: http://reports. weforum.org/human-capital-index-2013/ 
World Tourism Organization (2015). International Tourist Arrivals by Country of Destination, unwTo, vol 14. Statistical Annex. Recuperado de: http:// www.siimt.com/work/models/siimt/Resource/1de0bb9d-8199-48fbaf9a-769464f99875/PDF_RankingOMT_2015_May16.pdf

Zambon, S. (2016). Ten years after: The past, the present and the future of scholarly investigation on intangibles and intellectual capital (IC). Journal of Intellectual Capital, 17(1), 1-8. Recuperado de http://dx.doi. org/10.1108/JIC-11-2015-0093 